Case Report

\title{
Acquired Hemophilia B Through Liver Transplantation: A Case Report and Literature Review
}

\section{Christine Jane Kurian, Douglass Alan Drelich, Sanaa Rizk ${ }^{*}$}

Cardeza Foundation for Hematologic Research, Department of Medicine, Sidney Kimmel Medical College, Thomas Jefferson University, Philadelphia PA, USA

\section{Email address:}

sanaa.rizk@jefferson.edu (S. Rizk)

${ }^{*}$ Corresponding author

\section{To cite this article:}

Christine Jane Kurian, Douglass Alan Drelich, Sanaa Rizk. Acquired Hemophilia B Through Liver Transplantation: A Case Report and Literature Review. American Journal of Internal Medicine. Vol. 8, No. 6, 2020, pp. 312-315. doi: 10.11648/j.ajim.20200806.21

Received: October 4, 2020; Accepted: October 28, 2020; Published: December 16, 2020

\begin{abstract}
Hemophilia B, also called Christmas disease, is an X-linked inherited bleeding disorder that predominantly affects males, and it is caused by deficiency of coagulation factor IX. Factor IX is a coagulation factor produced in the liver. Liver transplantation from hemophilia B donors is neither widely documented nor performed due to presumed risk of developing increased bleeding tendency in the recipient due to potential acquired factor IX deficiency. In this review, we present a case of liver and kidney transplantation from a donor with mild hemophilia B to a recipient with no history of hemophilia B and literature review. This is a relatively rare situation with only two other prior case reports of acquired Hemophilia B through liver transplantation noted in 2015. The 2 cases presented in the literature lead to acquired hemophilia B in liver transplant recipients since donors were not screened due to mild disease and low suspicion level. In our case, the donor was known to have mild Hemophilia B and the recipient had a rapid decline - thus it is imperative to weigh risks and benefits of transplantation from this donor population. While those individuals with mild hemophilia B disease (and low bleeding burden) should be considered as an alternative donor for liver transplantation, appropriate counseling should be done with the recipient about possible bleeding risks and OLT should only occur after patient consent. A multidisciplinary approach should involve hematology in case factor replacement therapy is needed post transplantation and for long term follow up.
\end{abstract}

Keywords: Hemophilia B, Christmas Disease, Liver Transplant, Factor IX, Hemostasis

\section{Introduction}

Hemophilia B, also called Christmas disease, is an X-linked inherited bleeding disorder that predominantly affects males. Patients have factor IX deficiency due to a variation in the factor IX gene [1-3]. The prevalence of hemophilia B is estimated to be 1 in 25,000 to 30,000 males. Approximately half have severe disease (factor IX activity $<1$ percent of normal). Factor IX contributes to secondary hemostasis and formation of fibrin clot via intrinsic pathway leading to factor $\mathrm{x}$ activation. Clinical manifestations of hemophilia relate to bleeding from impaired hemostasis, sequelae from bleeding, or complications of coagulation factor infusion. Diagnostic criteria of Hemophilia B includes factor IX levels $<40 \%$ of normal and presence of a mutated F9 gene. Patients will have a characteristically prolonged partial thromboplastin time (PTT) that corrects on mixing studies.

Factor IX is a coagulation factor produced in the liver [4-8]. Liver transplantation from hemophilia B donors is neither widely documented nor performed due to presumed risk of developing increased bleeding tendency in the recipient due to potential acquired factor IX deficiency. In this review, we present a case of liver and kidney transplantation from a donor with mild hemophilia $\mathrm{B}$ to a recipient with no history of hemophilia B and literature review.

\section{Case}

The liver recipient was a 72-year-old male with a past medical history of decompensated alcoholic cirrhosis 
complicated by hepatocellular carcinoma, bladder carcinoma, prostate carcinoma, and immune thrombocytopenic purpura. His Model for End-Stage Liver Disease (MELD) score was 24 upon time of evaluation for transplant, and he was in acute renal failure, related to hepato-renal syndrome leading to hemodialysis. The patient was listed for liver-kidney transplantation with discussions to expand patient's pool of donors (US Centers for Disease Control and Prevention high-risk donors and hepatitis B core antibody-positive donors) due to rapidly progressive disease. Prior to transplantation, he did not have any history of recurring bleeding events (no Factor IX activity level prior to pre-transplant evaluation was available, however, PTT was normal at 34 seconds 5 years before presentation). His pre transplant labs are listed in table 1. Notably, factor IX activity was $38 \%$, likely due to his history of end stage liver disease.

Table 1. Pre-transplantation Lab values.

\begin{tabular}{lll}
\hline Lab & Reference Range & Pre-transplant value \\
\hline White blood cell count & $4-11 \mathrm{~B} / \mathrm{L}$ & 7.2 \\
Hemoglobin & $14-17 \mathrm{~g} / \mathrm{L}$ & 8.8 \\
Hematocrit & $42.0-52.0 \%$ & 24.8 \\
MCV & $80-99 \mathrm{fl}$ & 101 \\
Platelet count & $140-400 \mathrm{~B} / \mathrm{L}$ & 52,000 \\
PT & $8.9-13.1 \mathrm{~seconds}$ & 14.8 \\
INR & $0.81-1.19$ & 1.34 \\
PTT & $24-35 \mathrm{~seconds}$ & 44 \\
Factor IX level & $50-150 \%$ & $38 \%$ \\
Fibrinogen & $204-462 \mathrm{mg} / \mathrm{dL}$ & 192 \\
BUN & $12-27 \mathrm{mg} / \mathrm{dL}$ & 75 \\
Creatinine & $0.701 .4 \mathrm{mg} / \mathrm{dL}$ & 5.2 \\
eGFR & $>60 \mathrm{units}$ & 11 \\
Total bilirubin & $0.1-0.9 \mathrm{mg} / \mathrm{dL}$ & 0.8 \\
Direct bilirubin & $0-0.3 \mathrm{mg} / \mathrm{dL}$ & 0.3 \\
Alkaline phosphatase & $25-120 \mathrm{IU} / \mathrm{L}$ & 221 \\
AST & $7-42 \mathrm{IU} / \mathrm{L}$ & 46 \\
ALT & $<45 \mathrm{IU} / \mathrm{L}$ & 45 \\
\hline
\end{tabular}

The liver and kidney donor was a 38-year-old male patient who had congenital mild Factor IX deficiency. He did not have significant history of bleeding. He was not on any type of prophylactic factor replacement. He did not develop any factor IX inhibitor based on his records. No genotype testing was found in our records. He was admitted to the hospital for head trauma with massive intracranial bleed and became brain dead. The donor's factor IX level was $8 \%$ at the day of transplant.

We were consulted to discuss risk of bleeding and potential development of acquired Hemophilia B in the donor. Risks of transplantation with a donor with hemophilia B were thoroughly reviewed with the patient and it was understood that he may theoretically develop acquired hemophilia $\mathrm{B}$ requiring factor replacements therapy. As the patient's renal failure and liver failure were progressing, and weighing risks and benefits, he decided to accept the hemophilia B donor organs.

Orthotropic liver and kidney transplantation was performed on 3/6/2018. The hematology team closely followed him post-transplant. Following discussion, the decision was made to treat with standard half-life recombinant FIX product with the goal to maintain FIX troughs of $80-100 \%$ for the first 5 to 7 days, then troughs greater than 50\% afterward for 10-14 days, with peak levels not to exceed $200 \%$. His last factor IX replacement therapy was on $4 / 30 / 2018$. He was negative for factor IX inhibitor when tested post-op (AB titer $<0.6$ on $5 / 2 / 2018$ ). His factor IX levels and PTT post transplantation were as shown in Figure 1. His other factor activity levels were checked post-transplant, and also were normal, as seen in table 2 .

Table 2. Additional factor levels post-transplantation, on 3/12/2018.

\begin{tabular}{ll}
\hline Factor & Factor activity level (normal 50-150\%) post transplantation \\
\hline II & 58 \\
V & 114 \\
VII & 59 \\
VIII & 122 \\
X & 57 \\
\hline
\end{tabular}

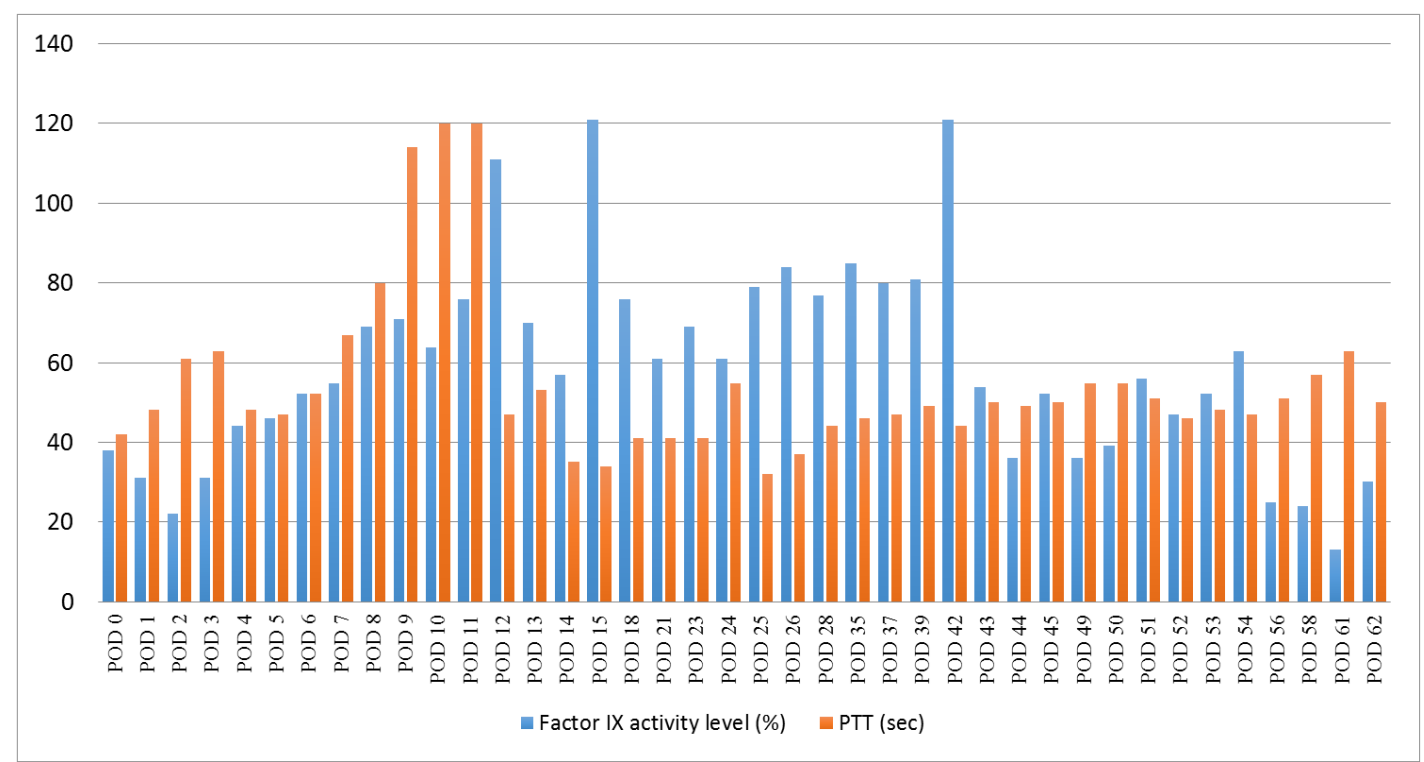

Figure 1. Trend of Factor IX levels and PTT values after transplantation. 
His postoperative course was complicated by many events. Immediately postoperatively, kidney graft function was delayed, requiring CVVHD. He had intermittent pneumatic compression for thromboprophylaxis for the first 2 days, then switched to heparin 5000 Units SQ Q 12 hours. On POD 6, he was found to have a femoral DVT and was placed on a heparin drip while receiving factor IX replacement. Our new target for factor IX trough level was to increase it to $70 \%$ to minimize bleeding events while on full dose anticoagulation, with peak not to exceed $200 \%$. At the time of diagnosis of his clot, his factor IX level was 52\%. Hemoglobin dropped on POD 11. Investigation revealed a peri-nephric hematoma, $7 \mathrm{~cm}$ in size. His anti-Xa level at the time was 0.23 , and Factor IX level was 57\%. Anticoagulation was stopped and an IVC filter was placed on POD 12. Evacuation of the hematoma was performed on POD 13. Embolization of the left L2, L3, L4 lumbar and left iliolumbar arteries was performed by interventional radiology on POD 16 and bleeding was controlled. Unfortunately, the patient developed Pseudomona pneumonia and developed hypoxic respiratory failure. His condition continued to deteriorate and his family made the decision to withdraw care on POD 55, and replacement factor stopped. His factor IX levels eventually dropped to around $20-30 \%$ in the following days before he passed away on POD 62.

\section{Discussion}

This is a relatively rare situation with only two other prior case reports of acquired Hemophilia B through liver transplantation noted in 2015 [9, 10]. The first report in literature was in 2015 by Bergstrom and colleagues who described an OLT in a 19 months old boy due to biliary atresia. He developed mild bleeding post OLT from vein puncture sites as well as bruising that led to work up showing mild hemophilia B with factor IX ranging from 5\%-10. His factor IX activity level was $8 \%$ with no inhibitor at time of evaluation. F9 gene sequencing on patient's leukocytes did not reveal any abnormality. However, gene sequencing of transplanted liver tissue revealed a F9 mutation. The donor was mildly symptomatic and not known to have a bleeding disorder as per his family members [9].

This was followed by another report in 2015 by Brunetta et al., who detailed a case of a new onset of FIX deficiency, in association with mild coagulation abnormalities following liver transplantation, attributable to an unrecognized hemophilia B in the donor. Active bleeding was noticed after a dental extraction performed 7 months after transplant. PTT was prolonged and factor IX activity was $11 \%$. In speaking with the donor's family, two thigh hematomas were noted once after a sports-related injury, but patient was never officially diagnosed with a bleeding disorder [10].

The hepatocyte is the primary cell type responsible for synthesizing factor IX, as shown in murine models [11]. Their studies showed that liver sinusoidal endothelial cells and extrahepatic cells (including the lung, spleen, kidney, brain, intestine, and tongue) did not express factor IX mRNA. In comparison, factor VIII is produced extrahepatically, and transplant of a liver from a donor carrying the diagnosis of hemophilia A did not lead to development of the bleeding disorder in the recipient [12-15].

There have been few reports of hemophilia B donors in liver transplantation. Patients with mild hemophilia frequently have a benign course and bleeding occurs only in response to surgery, tooth extractions, or major injuries. The diagnosis can be missed frequently if suspicion level is low. The 2 cases presented in the literature lead to acquired hemophilia B in recipients since donors were not screened due to mild disease and low suspicion level.

In our case, the donor was known to have mild Hemophilia $\mathrm{B}$ and the recipient had a rapid decline - thus it is imperative to weigh risks and benefits of transplantation from this donor population.

\section{Conclusion}

While those individuals with mild hemophilia B disease (and low bleeding burden) should be considered as an alternative donor for liver transplantation, appropriate counseling should be done with the recipient about possible bleeding risks and OLT should only occur after patient consent. A multidisciplinary approach should be done and it should involve hematology in case factor replacement therapy is needed post transplantation and for long term follow up.

\section{Conflict of Interest Disclosure}

The authors declare that they have no competing interests.

\section{Author Contribution Section}

All authors contributed in data collection and analysis, literature review and analysis as well as writing the paper.

\section{Essentials Section}

1. Liver transplantation from hemophilia B donors is not commonly performed due to the perceived risk of developing hemophilia $\mathrm{B}$ in the recipient.

2. We present a case of liver and kidney transplantation from a donor with mild hemophilia $\mathrm{B}$ to a recipient with no history of hemophilia B and literature review with development of mild hemophilia B in the recipient.

3. The hepatocyte is the primary cell type responsible for synthesizing factor IX, as shown in murine models. Their studies showed that liver sinusoidal endothelial cells and extrahepatic cells (including the lung, spleen, kidney, brain, intestine, and tongue) did not express factor IX mRNA.

4. While patients with mild hemophilia B disease should be considered as an alternative donor for liver transplantation, appropriate counseling should be done 
with the recipient about possible bleeding risks and OLT should occur after patient consent to it.

\section{References}

[1] "F9 coagulation factor IX [Homo sapiens (human)] - Gene NCBI.” Available at: https://www.ncbi.nlm.nih.gov/gene/2158. (Accessed: 24th February 2020).

[2] P. H. B. Bolton-Maggs and K. J. Pasi, "Haemophilias A and B," in Lancet, 2003, vol. 361, no. 9371, pp. 1801-1809, doi: 10.1016/S0140-6736(03)13405-8.

[3] K. Kurachi and E. W. Davie, "Isolation and characterization of a cDNA coding for human factor IX," Proc. Natl. Acad. Sci. U. S. A., vol. 79, no. 21 I, pp. 6461-6464, 1982, doi: 10.1073/pnas.79.21.6461.

[4] K. A. Boost et al., "Long-term production of major coagulation factors and inhibitors by primary human hepatocytes in vitro: Perspectives for clinical application," Liver Int., vol. 27, no. 6, pp. 832-844, Aug. 2007, doi: 10.1111/j.1478-3231.2007.01472.x.

[5] S. Kasuda et al., "Expression of coagulation factors from murine induced pluripotent stem cell-derived liver cells," Blood Coagul. Fibrinolysis, vol. 22, no. 4, pp. 271-279, Jun. 2011, doi: 10.1097/MBC.0b013e328344c63b.

[6] K. Ohashi, K. Tatsumi, R. Utoh, S. Takagi, M. Shima, and T. Okano, "Engineering liver tissues under the kidney capsule site provides therapeutic effects to hemophilia B mice," Cell Transplant., vol. 19, no. 6-7, pp. 807-813, Jun. 2010, doi: $10.3727 / 096368910 X 508924$.

[7] K. Tatsumi, K. Ohashi, M. Shima, Y. Nakajima, T. Okano, and A. Yoshioka, "Therapeutic effects of hepatocyte transplantation on hemophilia B," Transplantation, vol. 86, no. 1, pp. 167-170, Jul. 2008, doi: 10.1097/TP.0b013e31817b9160.
[8] K. Tatsumi et al., "Human Hepatocyte Propagation System in the Mouse Livers: Functional Maintenance of the Production of Coagulation and Anticoagulation Factors," Cell Transplant., vol. 21, no. 2-3, pp. 437-445, Mar. 2012, doi: $10.3727 / 096368911 \times 605349$.

[9] K. Bergstrom, A. Stevens, L. Srivaths, J. Economides, and D. L. Yee, "Haemophilia B acquired from liver transplantation: A case report and literature review," Haemophilia, vol. 21, no. 4. Blackwell Publishing Ltd, pp. e328--e330, Jul-2015, doi: 10.1111/hae.12699.

[10] D. M. Brunetta et al., "Hemophilia B acquired through liver transplantation," Liver Transplantation, vol. 22, no. 2. John Wiley and Sons Ltd, pp. 254-256, Feb-2016, doi: 10.1002/lt.24364.

[11] K. Tatsumi et al., "Hepatocyte is a Sole Cell Type Responsible for the Production of Coagulation Factor IX in Vivo," Cell Med., vol. 3, no. 1-3, pp. 25-31, Jan. 2012, doi: $10.3727 / 215517912 \times 639496$.

[12] D. Zanolini et al., "Extrahepatic sources of factor VIII potentially contribute to the coagulation cascade correcting the bleeding phenotype of mice with hemophilia A," Haematologica, vol. 100 , no. 7 , pp. 881-892, Jul. 2015, doi: 10.3324/haematol.2014.123117.

[13] C. L. Madeira et al., "Extrahepatic factor VIII production in transplant recipient of hemophilia donor liver.," Blood, vol. 113, no. 21, pp. 5364-5365, May 2009, doi: 10.1182/blood-2009-02-206979.

[14] T. Shahani et al., "Human liver sinusoidal endothelial cells but not hepatocytes contain factor VIII," J. Thromb. Haemost., vol. 12, no. 1, pp. 36-42, Jan. 2014, doi: 10.1111/jth.12412.

[15] C. J. Kurian, D. A. Drelich, and S. Rizk, "Successful liver transplant from a hemophilia A donor with no development of hemophilia A in recipient," J. Thromb. Haemost., vol. 18, no. 4, pp. 853-856, Apr. 2020, doi: 10.1111/jth.14750. 